\title{
Neutrinos from GRBs and their detection with ANTARES
}

\section{Tri L. Astraatmadja ${ }^{1,2}$ on behalf of the ANTARES Collaboration}

\author{
${ }^{1}$ Nikhef - National Institute for Subatomic Physics, \\ Science Park 1051098 XG Amsterdam, The Netherlands \\ ${ }^{2}$ LION - Leiden Institute of Physics, Leiden University, \\ PO Box 95042300 RA Leiden, The Netherlands \\ email: t.astraatmadja@nikhef.nl
}

\begin{abstract}
The detection principle of ANTARES and its sensitivity to GRB neutrinos will be discussed. Latest analysis of ANTARES data in coincidence with GRB direction and time of occurence will also be presented, as well as the prospects of neutrino detection with KM3NeT, the $\mathrm{km}^{3}$ neutrino telescope that will succeed ANTARES.
\end{abstract}

Keywords. astroparticle physics, gamma-ray burst: general, elementary particles

Most of our knowledge on the universe come from photons, and it is rightly so: they are abundantly produced, stable, and electrically neutral. However, photons interact electromagnetically with matter and thus could be quickly absorbed in matter. Other astrophysical carriers of information are cosmic rays, which are primarily protons accelerated to up to $10^{20} \mathrm{eV}$. Protons however are charged particles and are deflected by magnetic fields to a random direction, making it difficult to pinpoint their source. Neutrinos are electrically neutral and only interact with matter through the weak nuclear force. Thus they can travel through great distance and point back through the source.

High-energy neutrinos are produced from the interactions of protons with photons through the $\Delta^{+}$resonance, which produce neutral and charged mesons. The neutral mesons will then decay into gamma-rays while the charged mesons will decay into neutrinos. Calculating the neutrino energy spectrum based on the observed properties of the gamma-ray spectrum is commonly done using the formulation derived by Waxman \& Bahcall (1997).

The ANTARES neutrino telescope consists of a three-dimensional array of 885 lightsensitive photomultipliers (PMT). The PMTs are grouped into triplets, with 25 triplets forming one detector line. ANTARES has 12 such lines. The total instrumented volume of the detector is approximately $0.01 \mathrm{~km}^{3}$. It is located at a depth of $2475 \mathrm{~m}$ at the bottom of the Mediterranean Sea, $40 \mathrm{~km}$ offshore Toulon in the south of France.

When a neutrino passes through the Earth it will interact via the charged current interaction to produce a muon that would come out of the other side of the Earth. As it traverses the sea, the muon will produce Cherenkov light because its velocity is greater than the velocity of light in seawater. The PMTs that comprised ANTARES could detect these Cherenkov photons by recording the position of the PMT that got hit by a photon and the time of occurence. If we look for hits that are causally connected in space and time, we could accurately reconstruct the muon track.

Data coinciding with 40 GRBs in 2007 have been analysed. No neutrino event has been observed, but upper limit with $90 \%$ confidence level has been set. KM3NeT, the much larger successor of ANTARES, is currently being planned and is expected to have an instrumented volume of $1 \mathrm{~km}^{3}$. The first scientific data is anticipated in 2014 . 

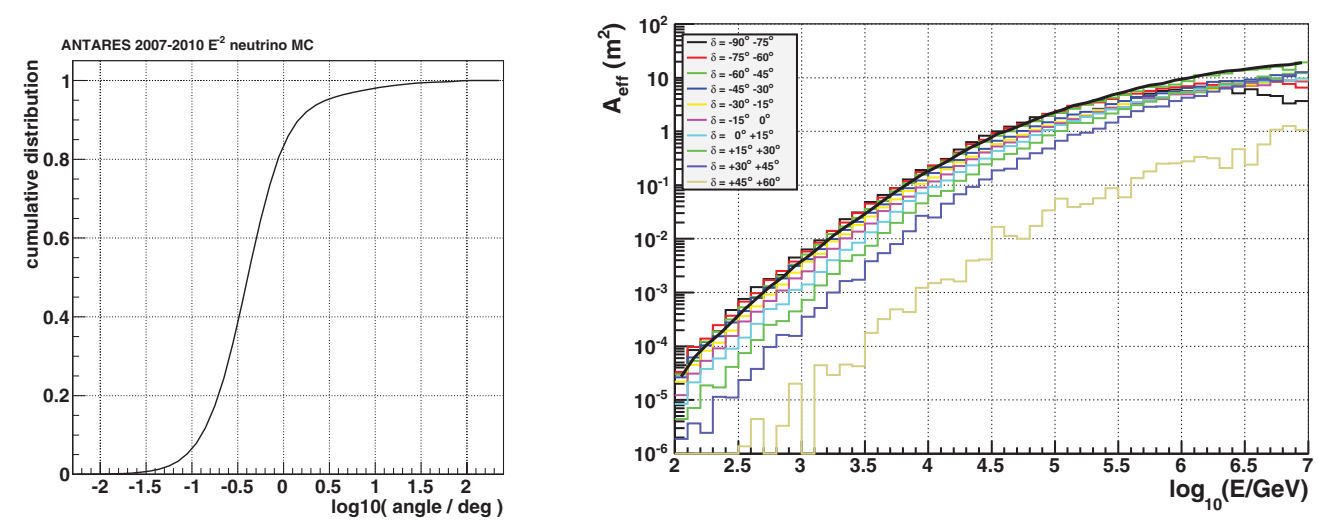

Figure 1. Left: Cumulative distribution of the angular error for $\epsilon_{\nu}^{-2}$ neutrino events that pass the quality cuts. The median angular error is $0.46^{\circ}$. Right: The neutrino effective area in 10 declination bands of 15 degrees as a function of neutrino energy, visibility included. The black line is zenith angle-averaged effective area.
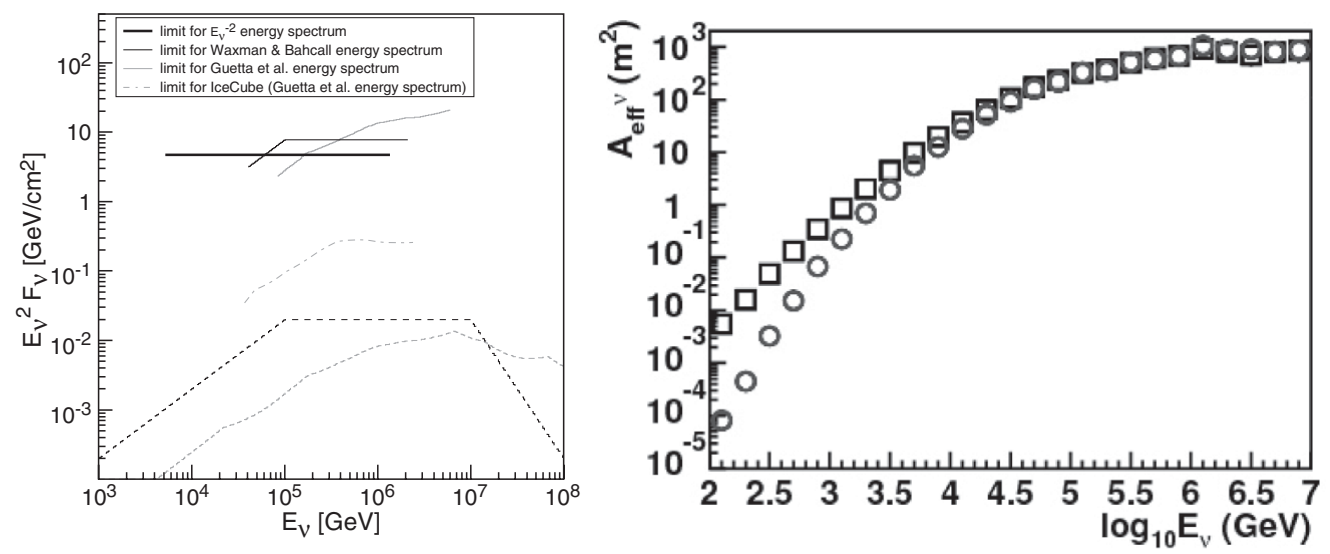

Figure 2. Left: Upper limits as a function of neutrino energy on the muon-(anti)neutrino fluence $F_{\nu}$ at $90 \%$ confidence level (CL), for 40 selected GRBs and for three different neutrino energy spectra. The total prompt emission duration of the 40 selected GRBs is $2114 \mathrm{~s}$. Four different limits are shown: limit for the $\epsilon_{\nu}^{-2}$ energy spectrum, the Waxman \& Bahcall (1999) energy spectrum, the Guetta et al. (2004) energy spectrum, and the IceCube 90\% CL upper limit from Abbasi et al. (2011). Right: Neutrino effective areas for the future KM3NeT detector. The plot is for upgoing neutrinos. The circles are after quality cuts optimised for searches for point-like neutrino sources and the squares are for looser quality cuts ensuring reasonable angular resolution.

\section{References}

Abbasi, R., et al. 2009, ApJ 701, L47

Abbasi, R., et al. 2011, Phys. Rev. Lett. 106, 141101

Aharonian, F., Buckley, J., Kifune, T., \& Sinnis, G. 2008, Rep. Prog. Phys. 71, 096901

Guetta, D., Hooper, D., Alvarez-Muñiz, J., Halzen, F., \& Reuveni, E. 2004, APh 20, 429

Waxman, E. \& Bahcall, J. 1997, Phys. Rev. Lett. 78, 2292

Waxman, E. \& Bahcall, J. 1999, Phys. Rev. D 59, 023002 